\title{
CIGRE 2020
}

\section{G. Christiner}

Online publiziert am 16. November 2020

(C) CIGRE - Reprint from www.cigre.org with kind permission 2020

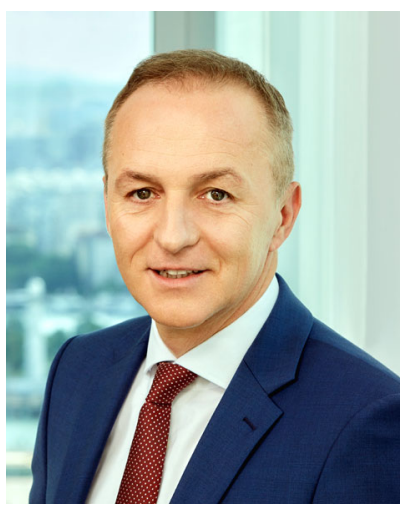

Dipl.-Ing. Mag. (FH) Gerhard Christiner
Die alle zwei Jahre in der letzten Augustwoche in Paris stattfindende CIGRE Session ist die weltweit bedeutendste Plattform ihrer Art. Sie bietet Vertretern der Energiewirtschaft und Industrie, ElektrotechnikExpert/innen und Wissenschaftler/innen die Gelegenheit zum Erfahrungsund Wissensaustausch.

Aufgrund der weltweiten COVID-19-Einschränkungen musste die diesjährige CIGRE Session als virtuelle Veranstaltung organisiert werden. Bei einem Event, das unter normalen Bedingungen über 3.500 Teilnehmer/innen eine Woche lang in Paris zusammenkommen lässt, ist dies keine einfache Aufgabe. Unter normalen Bedingungen erfordert die Organisation der CIGRE Session eine Vorbereitungszeit von mindestens zwölf Monaten. Das Organisationsteam hatte für die Umstellung vom geplanten Ablauf auf eine virtuelle eSession 2020 hingegen gerade einmal drei Monate zur Verfügung.

Trotz der knappen Vorbereitungszeit war die eSession 2020 ein großer Erfolg. Bereits in den letzten Jahren wurde CIGRE zu einer modernen Plattform für Expert/innen aus allen Bereichen der
Stromversorgung weiterentwickelt, die auch dem Wissenstransfer in strukturschwachen Ländern dienen soll. Diesem Ansatz folgend wurde - unterstützt durch die Weltbank - eine Afrika-Initiative gestartet, die bereits erste Früchte trägt und zuletzt auch zur Gründung von neuen Nationalkomitees führte. Weitere Schwerpunkte sind die konsequente Unterstützung von jungen Mitgliedern (CIGRE Next Generation Network - NGN) und von Frauen (CIGRE Women in Engineering).

CIGRE wurde 1921 gegründet und wird nächstes Jahr das 100jährige Jubiläum feiern. Zusammen mit dem durch COVID-19 ausgelösten Digitalisierungsschub ist CIGRE heute mehr als je zuvor ein weltweites digitales Netzwerk, das die Zukunft der Stromversorgung auf allen Ebenen aktiv mitgestaltet.

Die CIGRE eSession 2020 war geprägt von spannenden und fruchtbaren Fachdiskussionen. Insgesamt kamen rund 2.500 Delegierte aus rund 100 Ländern virtuell zur Diskussion aktueller Entwicklungen im Bereich der Energiewirtschaft zusammen und diskutierten aktuelle Themen der Stromversorgung. Einen Auszug daraus finden Sie, wie immer, in den folgenden Berichten.

Ich wünsche Ihnen eine spannende Lektüre!

Dipl.-Ing. Mag. (FH) Gerhard Christiner

Vorsitzender des Österreichischen Nationalkomitees der CIGRE

Hinweis des Verlags Der Verlag bleibt in Hinblick auf geografische Zuordnungen und Gebietsbezeichnungen in veröffentlichten Karten und Institutsadressen neutral. 